\title{
Performance Evaluation of Secure and Efficient AODV Routing Protocol using NS2
}

\author{
Meeta Singh \\ Research Scholar \\ Computer Science \& Engineering \\ Bhagwant University, Ajmer(Raj.)
}

\author{
Jigyasa Sharma \\ Post Doctoral Fellow \\ NIT Patna(Bihar)
}

\begin{abstract}
This paper focuses on the improvement on the performance of AODV single path routing protocol concerning the route maintenance mechanism with security. This paper presents the performance comparison of proposed protocol Secure and efficient AODV (SE-AODV) with AODV routing protocol using NS2. As the reactive routing protocols is completely different from the proactive routing protocols because they belong to two different routing families. Our proposed SEAODV routing protocol change the on-demand single path routing protocol into proactive multipath routing protocol in terms of predicting the link failure by utilizing the link state prediction algorithm and in terms of creating multiple routes by utilizing on-demand multipath routing approach.
\end{abstract}

\section{Keywords-}

Routing protocol, reactive, proactive, link failure, SE-AODV, AODV

\section{INTRODUCTION}

In MANET, nodes can communicate with each other without the intervention of any central controller. MANET is a collection of self organized nodes with transiver. MANET protocols are classified as: Proactive, Reactive and Hybrid. Research is going on these protocols.

Unobservable secure on-demand routing (USOR) protocol was proposed by W.Zhiguo et.al[1]. USOR cannot prevent the warmhole attacks. Huang.T et.al. [2] gives the backup routing scheme utilizing the 2-hop neighbor knowledge to establish backup paths. The proposed backup routing scheme was only used for single link breakup and not for multiple link breakups. Sesay S et.al. [3] presents the Adaptive load energy balancing that was combined with the hotspot mitigation scheme used only for dynamic system. C.David,et. al.[4] proposed a secure A-SAODV, they perform only some improvements on it. Chang J et. al. [5] have presented the power aware Ad hoc routing protocol, instead of the system as a whole there focus was based on individual nodes in the system. AODV based anycast protocol was proposed by W.Jianxi et. al.[6] but in the unevenly distributed of the traffic load it is not efficient.

A new extension protocol was proposed by us that standardizes the on-demand multipath and proactive routing approaches. In terms of the route request new mechanism works as on-demand multipath routing protocol; whereas in route maintenance it works as a proactive protocol. This can be endorsed by two reasons. Firstly, when the link to the next hop is broken, there was no proper route maintenance. Secondly, to evade sending route error packet to the source. This paper presents the performance analysis of proposed protocol Secure and efficient AODV routing protocol AODV) w.r.t. AODV in terms of varying node speed.

(SE-

To spread the traffic and to balance the network load, multipath routing approach provided with fault tolerance can be used. According to Pham and Perreau et. al. [7], the recent studies have shown that the uni-path routing approach has ghastly performance compared to the multipath routing approach in high density ad-hoc network. In single path routing approach, when a mobile node fails to send the data packet to the desired destination due to node mobility, a route error message is propagated and sent back to the source upon link failure indicating that the link is broken.

According to Nasipuri et. al. [8], the use of multipath routing approach in AODV protocol keeps long time the connection from the source to the destination compared to the single path approach. Therefore, maintaining multiple paths for every source-destination pair would reduce the route discovery process cost, balance the traffic more equally across the network and alleviate the effect caused by congested links. According to Nasipuri et al. in [9], for providing quality of service in mobile ad-hoc networks multipath routing approach is more assuring because multipath routing approach can provide load balancing, fault tolerant and higher throughput.

\section{DESIGN AIM OF SE-AODV ROUTING PROTOCOL}

To improve the performance of the current protocol two approaches are used. In the first approach the route maintenance is based on the proactive nature to predict the link breakage in advance by utilizing the link state prediction algorithm. In the second approach, multipath routing is used to discover and divert the data packet over multiple disjoint routes without sending a warning message with secure transmission to the source node. The occurrence of link failure is reduced with the help of SE-AODV protocol concept, consequently leads to reduce the route discovery process.SEAAODV is implemented on NS2. The NS2 simulations have two sets of scenario files:

- Movement generator pattern file

- $\quad$ Traffic generation pattern file

In the movement scenario file the simulation area and the mobility model of randomly distributed mobile nodes over the simulation time period are defined bu using Setdest tool and in the traffic pattern file, the characteristics of data communications, data packet size, packet type, packet transmission rate and the number of traffic flows are defined. The traffic files is generated using cbrgen utility of NS2. 


\section{SIMULATION SETUP}

One of the main constraints on mobile nodes in mobile ad-hoc network is the route enabling when the nodes keep moving. To study the performance of routing protocols under such conditions, it needs to consider the mobility patterns of entire network. Thus, this section evaluates the effects of node mobility on the performance of SE-AODV and AODV routing protocols using NS2, different maximum node speeds in the network have been considered. The node speeds are ranging as follows $10,20,30,40$ and $50 \mathrm{~m} / \mathrm{s}$, while the rest of the parameters are fixed. Each simulation consists of 50 mobile nodes placed over a simulation area of $1000 \mathrm{~m} \mathrm{x}$ $1000 \mathrm{~m}$. The area specified is demonstrating a square space in order to allow 50 mobile nodes to move inside. A square area does not discriminate one direction of motion like a rectangular area does. Network stability is indirectly proportional to node speed i.e. network is stable only if the node speed is low \& vice-versa.

Table 1: Simulation Parameters

\begin{tabular}{|l|l|}
\hline Parameters & Values \\
\hline No. of nodes & 50 \\
\hline Terrain size & $1000 \mathrm{~m} \mathrm{x} 1000 \mathrm{~m}$ \\
\hline Max-speed & $10,20,30,40,50 \mathrm{~m} / \mathrm{s}$ \\
\hline Pause time & $0 \mathrm{sec}$ \\
\hline Simulation time & $1000 \mathrm{sec}$ \\
\hline Traffic type & $\mathrm{CBR}$ \\
\hline Packet rate & $10 \mathrm{pkt} / \mathrm{sec}$ \\
\hline Packet size & $512 \mathrm{bytes}$ \\
\hline Transmission range & $250 \mathrm{~m}$ \\
\hline Movement model & Random waypoint \\
\hline MAC layer protocol & IEEE 802.11 \\
\hline Omni-directional & Antenna \\
\hline
\end{tabular}

\section{RESULT AND ANALYSIS}

The node speed has become an essential parameter in MANET to monitor the performance of the routing protocols. This section is observed that when the node speed decreases, the network topology is more stable, and both routing protocols perform well. However, when the node speed increases, SE-AODV protocol outperforms AODV protocol in terms of average end-to-end delay, higher packets delivery ratio, lower normalized control overhead, and lower routing overhead. The node speed plays an important role in determining the performance of the routing protocol which imposes a stress to the ad-hoc routing protocols due to the link failure and consequent re-initiate the route discovery mechanism. The link failure is caused by invalid routes (mobile nodes move out of the transmission range) or stale routes (mobile nodes with expired routes). When the link failure occurs, the routing control messages (RREQ, RREP, and RRER) also increase.

The accumulation of retransmission data packets increase the network load which results in less packet delivery ratio and more routing overhead. For the AODV results with respect of varying node speed, the probability of getting a stale route in its cache is very high. As a result, AODV in all the scenarios of the node speed parameter shows that AODV performs poorly compared to SE-AODV. AODV replies heavily on the route cache to determine a valid route which is prone to be stale with the increase of node speed. However, selecting stale routes from the cache of AODV protocol causes two problems: i. Consumption of additional network bandwidth and interface queue slots even though the packet is eventually dropped.

ii. Possible pollution of caches in other mobile nodes. This upholds AODV to work well in low mobility and small network size.

One of the factors of SE-AODV's advance is by providing the routing table to store the node disjoint routes to destination and supports multipath routing approach. Therefore, SEAODV establishes routes quickly and minimizing the communication overhead by its routing maintenance algorithms reaction to the topological changes. In all scenarios where the node speed presents, SE-AODV protocol is performed well. On the other hand, AODV causes more packet drops and high delay than SE-AODV routing protocol. The simulation results show that the node speed affects the performance of SE-AODV and AODV protocol differently. In the presence of high mobility, the link failures can occur more frequently. The link failure in AODV protocol initiates a new route discovery process due to the limited alternative routes in the route cache. Thus, the cost of the route discoveries in AODV protocol is directly proportional to the number of route breakages. The reaction of AODV to the link failures in comparison is different from SE-AODV protocol and causes route discovery more often because of the abundance of cached routes at each mobile node. Thus, the route discovery is delayed in AODV until all learned routes break. However, with high mobility environment, the chances of the cache routes being invalid are quite high in AODV protocol.

This section is observed that SE-AODV protocol has lower route re-discovery process than AODV. The reason is that the SE-AODV protocol finds more stable route during the route maintenance process by providing multiple routes to the destination, and maintain the route proactively. Hence, the number of initiating a route discovery process in SE-AODV protocol is reduced in high mobility environment. Tables 2 and 3 show the average data results for both protocols with respect of varying node speed parameter.

Table 2: Average Data for AODV with Respect of Varying Node Speed

\begin{tabular}{|l|l|l|l|l|l|}
\hline $\begin{array}{l}\text { Node } \\
\text { Speed / } \\
\text { Metrics }\end{array}$ & $\begin{array}{l}\mathbf{S 1 - 1 0} \\
\mathbf{m} / \mathbf{s}\end{array}$ & $\begin{array}{l}\text { S2-20 } \\
\mathbf{m} / \mathbf{s}\end{array}$ & $\begin{array}{l}\mathbf{S 3 - 3 0} \\
\mathbf{m} / \mathbf{s}\end{array}$ & $\begin{array}{l}\mathbf{S 4 - 4 0} \\
\mathbf{m} / \mathbf{s}\end{array}$ & $\begin{array}{l}\mathbf{S 5 - 5 0} \\
\mathbf{m} / \mathbf{s}\end{array}$ \\
\hline $\begin{array}{l}\text { Overhea } \\
\mathrm{d}\end{array}$ & 2634 & 9381 & 12745 & 16093 & 22718 \\
\hline PDR & $\begin{array}{l}88.68 \\
\%\end{array}$ & $\begin{array}{l}83.60 \\
\%\end{array}$ & $\begin{array}{l}80.43 \\
\%\end{array}$ & $\begin{array}{l}77.11 \\
\%\end{array}$ & $\begin{array}{l}75.20 \\
\%\end{array}$ \\
\hline NRL & 0.52 & 1.37 & 1.84 & 2.26 & 3.47 \\
\hline Delay & $0.099 \mathrm{~s}$ & $0.303 \mathrm{~s}$ & $0.398 \mathrm{~s}$ & $0.718 \mathrm{~s}$ & $0.956 \mathrm{~s}$ \\
\hline $\begin{array}{l}\text { Packet } \\
\text { Drop }\end{array}$ & 1450 & 1928 & 2322 & 2578 & 2932 \\
\hline
\end{tabular}

Table 3: Average Data for SE-AODV with Respect of Varying Node Speed

\begin{tabular}{|l|l|l|l|l|l|}
\hline $\begin{array}{l}\text { Node } \\
\text { Speed / } \\
\text { Metrics }\end{array}$ & $\begin{array}{l}\mathbf{S 1 - 1 0} \\
\mathbf{m} / \mathbf{s}\end{array}$ & $\begin{array}{l}\mathbf{S 2 - 2 0} \\
\mathbf{m} / \mathbf{s}\end{array}$ & $\begin{array}{l}\mathbf{S 3 - 3 0} \\
\mathbf{m} / \mathbf{s}\end{array}$ & $\begin{array}{l}\mathbf{S 4 - 4 0} \\
\mathbf{m} / \mathbf{s}\end{array}$ & $\begin{array}{l}\mathbf{S 5 - 5 0} \\
\mathbf{m} / \mathbf{s}\end{array}$ \\
\hline $\begin{array}{l}\text { Overhea } \\
\text { d }\end{array}$ & $\begin{array}{l}1236.6 \\
6\end{array}$ & $\begin{array}{l}2841.6 \\
6\end{array}$ & $\begin{array}{l}6143.3 \\
3\end{array}$ & $\begin{array}{l}8328.3 \\
3\end{array}$ & 12140 \\
\hline
\end{tabular}




\begin{tabular}{|l|l|l|l|l|l|}
\hline PDR & $99.08 \%$ & $96.55 \%$ & $94.26 \%$ & $93.08 \%$ & $\begin{array}{l}91.03 \\
\%\end{array}$ \\
\hline NRL & 0.26 & 0.60 & 0.87 & 0.92 & 1.33 \\
\hline Delay & $0.017 \mathrm{~s}$ & $0.033 \mathrm{~s}$ & $0.060 \mathrm{~s}$ & $0.107 \mathrm{~s}$ & $0.152 \mathrm{~s}$ \\
\hline $\begin{array}{l}\text { Packet } \\
\text { Drop }\end{array}$ & 510 & 731.31 & $\begin{array}{l}1014.1 \\
6\end{array}$ & $\begin{array}{l}1205.8 \\
3\end{array}$ & 1367 \\
\hline
\end{tabular}

\subsection{Routing Overhead vs. Node Speed}

The routing overhead is the cost to build and maintain the routing path. The routing overhead generated by SE-AODV and AODV protocols increases as the node speed increases. The AODV protocol has low performance for routing overhead compared to SE-AODV performance. Because in high mobility network condition, there is possibility that more link failure occurs for AODV protocol. When the node speed increases, the network topology changes frequently is also increased, and can trigger more new route discovery process which results more link break. The link break leads to increase the number of route request packets that are generated and distributed in the network. On the other hand, SE-AODV protocol reduces the frequency of the route rediscovery in high mobility environment by recovering the link quickly and diverts the data packets into a stable route.

The result of routing overhead for AODV protocol in the first scenario S1-10 is 2634 where the SE-AODV result is 1236.66. The result of the first, the network has been stable, which means the link failure does not happen frequently. In such situation, AODV protocol incurs not much overhead compared to SE-AODV protocol in the first scenario. The reason because the use of aggressive route cache in AODV protocol that can cache learn routes from the route discovery which can reduce frequent broadcasting route request messages to the destination in low mobility environment. However, the values of routing overhead increases more compared to SE-AODV as the node movement increases.

For instance, the values of the routing overhead for SEAODV are S2-20 m/s (2841.66), S3-30 (6143.33), S4-40 m/s (8328.33) and S5-50 m/s (12140). Where AODV protocol are $\mathrm{S} 2-20 \mathrm{~m} / \mathrm{s}$ (9381), S3-30 m/s (12745), S4-40 m/s (16093), and S5-50 (22718).

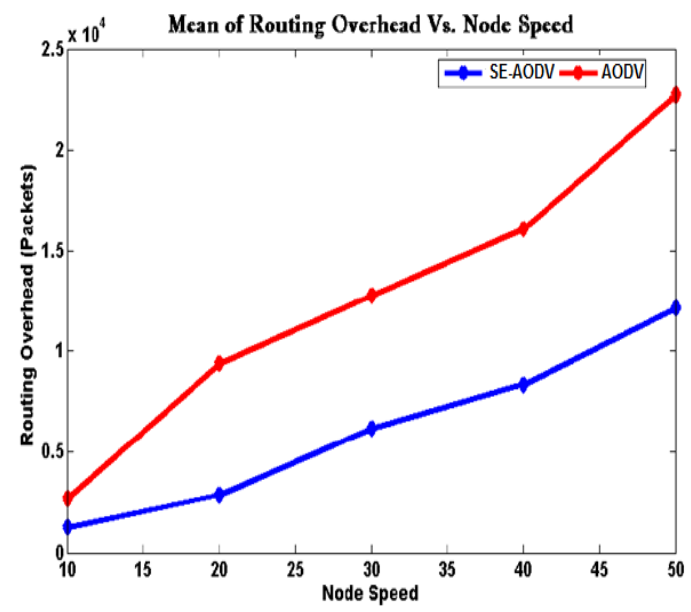

Figure 1: Routing Overhead vs. Node Speed

\subsection{Packet Delivery Ratio vs. Node Speed}

The performance of SE-AODV and AODV protocols is evaluated in terms of the packet delivery ratio against varying node speeds. In the situations of low mobility, the results values of packet delivery ratio are high which means the packet drop is less, and indicates the routing overhead is less as well. This is because the packet drop performance depends on the number of packets that are delivered at the destination node, whereas the routing overhead depends on the network congestion. Therefore, the packet delivery ratio is an important metric among quantitative metrics that can be used for evaluating the performance of a routing protocol in mobile ad-hoc network. In order to understand how the node speed affects the routing protocol performance, the node speed is varying in each scenario while the rest of parameters are maintained constant. SE-AODV protocol uses more stable paths and also sends the data packets from multiple paths, so it will able to deliver more data from the source to the desired destination even in highly dynamic network. On the other hand, the performance of AODV protocol decreases in high mobility network. When the speed of mobile node increases, the probability of link failure increases. Thus the number of packet drops also increases. SE-AODV protocol has higher packet delivery ratio than AODV protocol in all scenarios. The values of PDR for SE-AODV are S1-10 m/s (99.08\%), $\mathrm{S} 2-20 \mathrm{~m} / \mathrm{s} \quad(96.55 \%), \quad \mathrm{S} 3-30 \mathrm{~m} / \mathrm{s} \quad(94.26 \%), \quad \mathrm{S} 4-40 \mathrm{~m} / \mathrm{s}$ $(93.08 \%)$ and $\mathrm{S} 5-50 \mathrm{~m} / \mathrm{s}(91.03 \%)$. Where the values of PDR for AODV protocol are $\mathrm{S} 1-10 \mathrm{~m} / \mathrm{s}(88.68 \%), \quad \mathrm{S} 2-20 \mathrm{~m} / \mathrm{s}$ $(83.60 \%), \mathrm{S} 3-30 \mathrm{~m} / \mathrm{s}(80.43 \%), \mathrm{S} 4-40 \mathrm{~m} / \mathrm{s}(77.11 \%)$ and $\mathrm{S} 5-$ 50 $(75.20 \%)$.

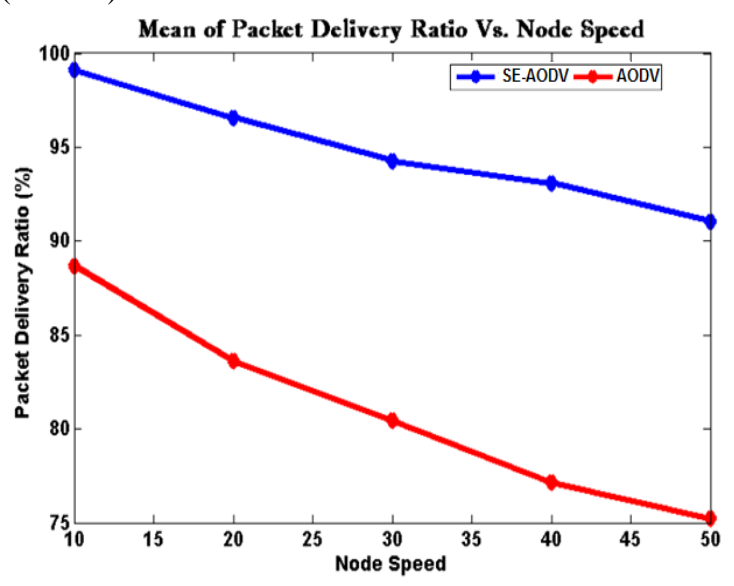

Figure 2: Packet Delivery Ratio vs. Node Speed

\subsection{Normalized Routing Load vs. Node Speed}

NRL identifies the number of routing control messages that can be conveyed per data packet delivered at the destination. In NRL, it is enough to find out the efficiency and scalability of the routing protocol's performance. Figure 3 shows that the normalized routing load increases whenever the node speed increased. In addition, there will be more link failure when the mobile nodes move with high speed which leads to increase the control packets (RREQ, RREP, and RRER). However, the routing protocol that transmitted a large number of control packets can increase the probability of packets collision and it also increase the waiting time of data packets in transmission buffering queue. In the first scenario $(\mathrm{S} 1-10 \mathrm{~m} / \mathrm{s})$ for SEAODV and AODV protocols where the number of routing packets conveyed per data packets in this moment are less compared with other scenarios. The values of NRL in the 
scenario $\mathrm{S} 1-10 \mathrm{~m} / \mathrm{s}$ for SE-AODV in are 0.26 , whereas for AODV protocol in the same scenario is 0.52 . The next value of NRL for SE-AODV in S2-20 m/s are $(0.60), S 3-30 \mathrm{~m} / \mathrm{s}$ $(0.87), \mathrm{S} 4-40 \mathrm{~m} / \mathrm{s}(0.92)$ and $\mathrm{S} 5-50 \mathrm{~m} / \mathrm{s}(1.20)$. Whereas for AODV are $\mathrm{S} 2-20 \mathrm{~m} / \mathrm{s}$ (1.37), S3-30 m/s (1.84), S4-40 m/s (2.26) and S5-50 m/s (3.47). The values of NRL for both protocol in the scenarios $S 2-20 \mathrm{~m} / \mathrm{s}, \mathrm{S} 3-30 \mathrm{~m} / \mathrm{s}, \mathrm{S} 4-40 \mathrm{~m} / \mathrm{s}$ are higher than the previous one $(\mathrm{S} 1-10 \mathrm{~m} / \mathrm{s})$. It indicates that the network condition was changed where the total number of routing packets sent is more compared to the first scenario $(\mathrm{S} 1-10 \mathrm{~m} / \mathrm{s})$ and also the total data packets received is less than the first scenario $(\mathrm{S} 1-10 \mathrm{~m} / \mathrm{s})$. The simulation results show that the SE-AODV protocol outperforms AODV protocol in terms of normalized routing load.

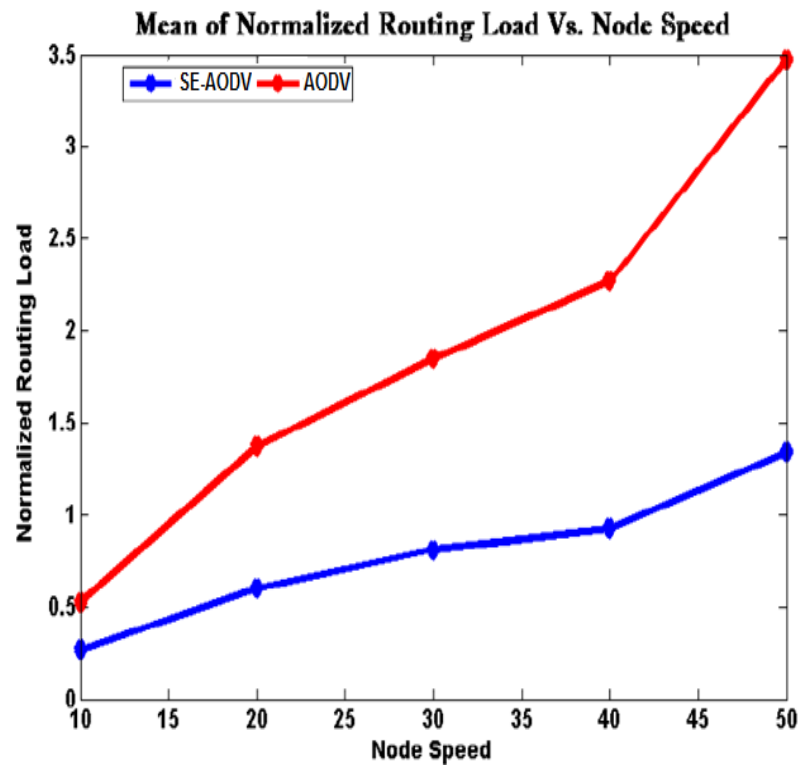

Figure 3: Normalized Routing Load vs. Node Speed

\subsection{Average End-to-End Delay vs. Node Speed}

The average end-to-end delay calculates the time of packet delivery at the destination subtract the time of packets transmitted and divided by the number of data packets received.

The average end-to-end delay of SE-AODV protocol is lower than AODV due to the availability of multiple routes during the data transmission in SE-AODV which avoids the route discovery latency that contributes to the delay when an active route predict to be failed. Moreover, the source node distributes the data packets through multiple different routes in order to manage the congestion, thereby reducing the queue waiting time of the data packets. On the other hand, the average end-to-end delay increases in AODV protocol because of the resurrection of data packets at the source nodes when the MAC-managed transmissions fail.

The average end-to-end delays of SE-AODV and AODV protocols in all scenarios increases, but the delay of SEAODV is less compared to the delay of AODV. When the mobility is high in AODV protocol, the routes in the cache are being stale. Eventually, when the route discovery is started, the large numbers of replies received in response are connected with high MAC overhead, and cause more interference to the data traffic. Thus, the cache staleness issue and high MAC overhead together lead to decrease the performance of AODV protocol in high mobility scenarios.
Moreover, if there is no route available in the route cache of the intermediate nodes, the delay will increase due to difficulty of finding a new route to divert the data packets. All these will lead to increase the average-end-to-end delay.

The first scenario results $(\mathrm{S} 1-10 \mathrm{~m} / \mathrm{s})$ for both protocols has shown a low end-to-end delay for SE-AODV is (0.017 s) and for AODV is $(0.099 \mathrm{~s})$. In the first scenario, the delay of AODV protocol shows better compared to the next scenarios result. This is because in case the link failure happens, the intermediate node able to find an alternative route from its route cache which avoids sends a route error message to the source node. The results of the delay in the next scenarios are increased. For instance, for SE-AODV the delays are S2-20 $\mathrm{m} / \mathrm{s}(0.033 \mathrm{~s}), \mathrm{S} 3-30 \mathrm{~m} / \mathrm{s}(0.060 \mathrm{~s}), \mathrm{S} 4-40 \mathrm{~m} / \mathrm{s}(0.107 \mathrm{~s})$ and $\mathrm{S} 5-50 \mathrm{~m} / \mathrm{s}(0.152 \mathrm{~s})$. Whereas for AODV protocol, the delays are $\mathrm{S} 2-20 \mathrm{~m} / \mathrm{s}(0.303 \mathrm{~s}), \mathrm{S} 3-30 \mathrm{~m} / \mathrm{s}(0.398 \mathrm{~s}), \mathrm{S} 4-40 \mathrm{~m} / \mathrm{s}$ $(0.718 \mathrm{~s})$ and $\mathrm{S} 5-50 \mathrm{~m} / \mathrm{s}(0.956 \mathrm{~s})$.

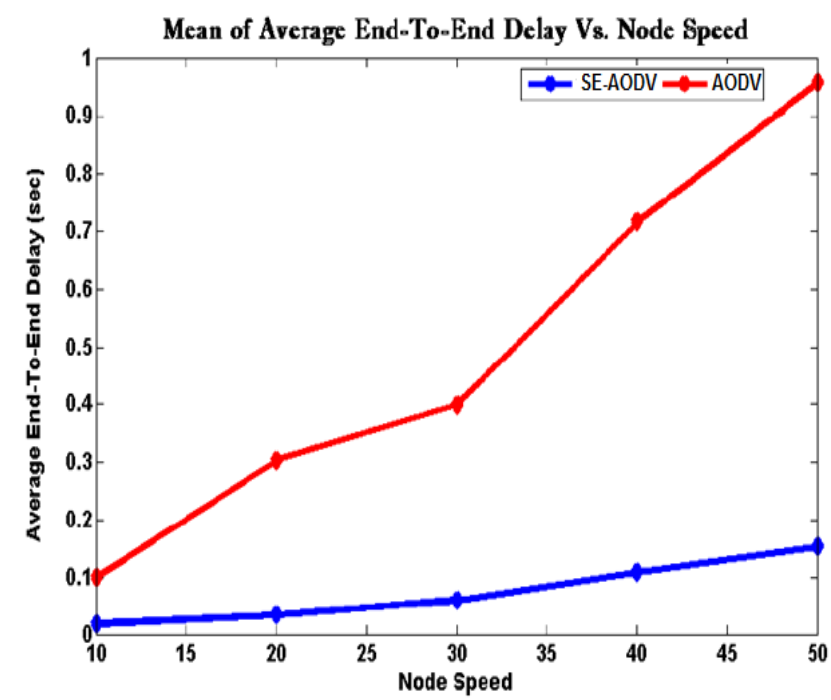

Figure 4: Average End-to-End Delay vs. Node Speed

\subsection{Packet Drop vs. Number of Nodes}

According to Kumar in [10], the high speed indicates more packet loss, increase the end-to-end delay, and increase the congestion in the network. The reason is because the routing protocol is sending route request packets on a broken route that is still considers being valid and thus packets in the node buffers are dropped due to the congestion and timeout. The backup route in AODV protocol is intersecting and the link failure is likely to be public node of two routes. Hence, the result of the link break leads to larger failure probability of the backup path restoration and further cause a higher loss probability of data packet. However, SE-AODV protocol can rapidly recover the link before it disconnected and diverts the data packets into a new disjoint route to forward to the destination. Moreover, the source node will initiate the route discovery only when all the discovered routes fail.

The first scenario when the node speed is $10 \mathrm{~m} / \mathrm{s}$ has shown low packets are dropped for SE-AODV is 510 and for AODV is 1450 . The results of packet drop are increased as the node speed increases in the next scenarios. For instance, for SEAODV the packet drop are S2-20 m/s (731.31), S3-30 m/s (1014.16), S4-40 m/s (1205.83) and $\mathrm{S} 5-50 \mathrm{~m} / \mathrm{s}$ (1367). Whereas for AODV protocol, the packet drops are $\mathrm{S} 2-20 \mathrm{~m} / \mathrm{s}$ (1928), S3-30 m/s (2322), S4-40 m/s (2578) and S5-50 m/s (2932). 


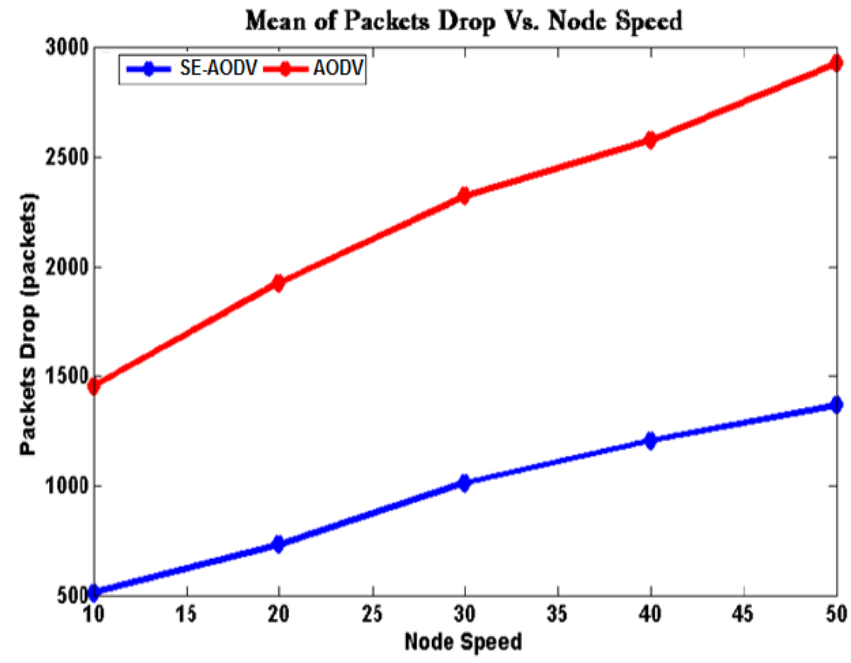

Figure 5: Packet Drop vs. Node Speed

\section{CONCLUSION}

The node speed has become an essential parameter in MANET to monitor the performance of the routing protocols. This section is observed that when the node speed decreases, the network topology is more stable, and both routing protocols performs well. However, when the node speed increases, SE-AODV protocol outperforms AODV protocol in terms of average end-to-end delay, higher packets delivery ratio, lower normalized control overhead, and lower routing overhead. The node speed plays an important role in determining the performance of the routing protocol which imposes a stress to the ad-hoc routing protocols due to the link failure and consequent re-initiate the route discovery mechanism. The link failure is caused by invalid routes (mobile nodes move out of the transmission range) or stale routes (mobile nodes with expired routes). When the link failure occurs, the routing control messages (RREQ, RREP, and RRER) also increase.

The accumulation of retransmission data packets increase the network load which results in less packet delivery ratio and more routing overhead. For the AODV results with respect of varying node speed, the probability of getting a stale route in its cache is very high. As a result, AODV in all the scenarios of the node speed parameter shows that AODV performs poorly compared to SE-AODV. AODV replies heavily on the route cache to determine a valid route which is prone to be stale with the increase of node speed.

\section{REFERENCES}

[1] W.Zhinguo et. el., "USRO:An Unobservable Secure OnDemand Routing Prot ocol for Mobile ad hoc networks", in proceedings of IEEE transactions on wireless communication, 2012.

[2]Huang Tsung-Chuan; Huang, Sheng-Yu; Tang, Lung," AODV-Based Backup Routing Scheme in Mobile Ad Hoc Networks", in proc. IEEE International Conference on Communications and Mobile Computing (CMC), Vol. 3, Page(s): $254-258,2010$.

[3]Sesay,S; Yang,Z.K; He,J.H; Xiang,J ; Du,X , “ Ensuring efficiency and robustness in MANET" in proc. IEEE International Conference on Mobile Technology, Applications and Systems, Page: 6, 2005

[4]C.Davide et al., "Securing AODV:The A-SAODV Secure routing protocol", published in IEEE Communication Magazine, 2008.

[5]Chang. J and Tassiulas. L, "Energy conserving routing in wireless adhoc networks," in proc. IEEE International Conference on "IEEE Computer and Communications Societies, INFOCOM”, Vol.1, Page(s): 22 - 31, 2000.

[6] W. Jianxin et. el. , "An AODV based anycast protocol in Mobile Ad hoc networks", in proceedings of IEEE International symposium on Personal, indoor \& Mobile communication, 2003.

[7] Pham, P. and S. Perreau, " Multi-path Routing Protocol with Load Balancing Policy in Mobile Ad Hoc Network", Fourth IFIP International Conference on Mobile and Wireless Communications Networks 2002, Sept 2002

[8] Nasipuri A, Castaneda R, Das SR. "Performance of multipath routing for on-demand protocols in mobile ad hoc networks", ACM/Kluwer Mobile Networks and Applications (MONET) 2001; 6(4): 339-349.

[9] N. Jain, S. Das, and A. Nasipuri. "A Multichannel CSMA MAC protocol with Receiver-Based Channel Selection for MultiHop Wireless Networks", IEEE IC3N, Phoenix, October 2001

[10] V. Kumar, "Simulation and Comparsion of AODV and DSR Routing Protocols in MANET," Master's thesis, Dept. of Computer Science and Engineering,Thapar University, 2009 